without pulmonary AVMs, for a series of noninvasive and invasive investigations, using an unbiased online survey.

Results Of 677 HHT-affected participants, 284 reported performing forced expiratory manoeuvres either with or without a noseclip in place. The median age was 54 years (interqurtile range 44-62) and the majority were female. All described nosebleeds: $130(46 \%)$ experienced these at least daily, and a further $95(33 \%)$ at least once per week. Only 1 of $253(0.4 \%)$ reported nosebleeds were worse after finger oximetry measurements, compared to 53/282 (18.8\%) after "blowing out hard without a noseclip" (chi-squared p $<0.0001$ ). A higher proportion still reported nosebleeds were worse when a noseclip was used (66/ 192 (34.3\%, p = 0.0003)). Similarly, migraine headaches (which are more frequent in people with HHT), were reported to be worse after forced expiratory manoeuvres both with (10/85 $(11.7 \%)$ and without $(11 / 105(10.5 \%))$ a noseclip, but not after oximetry or being weighed.

Conclusion Noseclip use should be restricted in people already experiencing regular nosebleeds, and further pretest information may be required.

\section{P34 LONG TERM OUTCOMES FOR PATIENTS WITH PULMONARY ARTERIOVENOUS MALFORMATIONS CONSIDERED FOR LUNG TRANSPLANTATION}

${ }^{1} \mathrm{CL}$ Shovlin, ${ }^{1} \mathrm{JMB}$ Hughes, ${ }^{2} \mathrm{M}$ Layton, ${ }^{1} \mathrm{E}$ Boother, ${ }^{1} \mathrm{DJ}$ Allison, ${ }^{2} \mathrm{JE}$ Jackson. ${ }^{1} / \mathrm{mperial}$

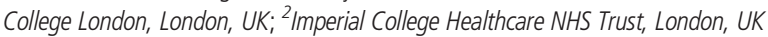

\subsection{6/thoraxjnl-2016-209333.177}

Introduction and objectives Pulmonary arteriovenous malformations (PAVMs) are abnormal vessels that provide anatomic rightto-left shunts, and frequently result in severe hypoxaemia. Lung transplantation is sometimes considered if PAVMs are not amenable to treatment by embolization or surgical resection.

Methods A retrospective review was performed of patients with PAVMs assessed between 1999 and 2016 at a single UK institution. Characteristics of those considered and referred for lung transplantation assessment were examined.

Results Between May 1999 and July 2016, 707 patients with PAVMs were reviewed. Six were or had been formally considered for lung transplantation purely for PAVMs, 4 as adults ( 3 at our institution between 1989-1995), 2 as children. Ages ranged from 2-47 (median 22) years. Four were from the UK, two had been assessed in non UK countries. All had hereditary haemorrhagic telangiectasia (HHT). Three had suffered a cerebral abscess due to their PAVMs. The adults had undergone maximal embolisation of PAVMs, and the children were considered to have untreatable disease. The median $\mathrm{SaO}^{2}$ was $79 \%$ on air.

One adult underwent lung transplantation overseas and died peri-operatively. The other five were not transplanted, in four cases at the patients' request. Currently, their survival post assessment for lung transplant ranges from 15 to 27 (median 22) years, with four of the five still alive.

All five maintained arterial oxygen content by marked secondary erythrocytotic responses when not iron deficient. Four had subsequent embolisation treatments. One had three successful pregnancies, and another is working full time with no supplementary oxygen. However, two had a cerebral abscess (one fatal); three had deep vein thromboses; two experienced long term disabling pain attributed to hypertrophic pulmonary osteoarthropathy in one; and three have exercise limitation requiring supplementary oxygen. Antiplatelet therapy was recommended for two due to ischaemic strokes; one remains anticoagulated 16 years after the insertion of a caval filter; and one is regularly evaluated for hyperviscosity symptoms that would precipitate isovolaemic venesection.

Conclusion Very long term stability is evident in the five patients who did not receive a lung transplant. The cohort remain at high risk of cerebral abscess and other complications of right-to-left shunting.

\section{P35 A RETROSPECTIVE OBSERVATIONAL STUDY DESIGNED TO CHARACTERISE INDIVIDUALS WITH PULMONARY ARTERIOVENOUS MALFORMATIONS (PAVMS) AND CEREBRAL ABSCESSES AT A SINGLE INSTITUTION}

${ }^{1} \mathrm{EJ}$ Boother, ${ }^{2} \mathrm{~S}$ Brownlow, ${ }^{2} \mathrm{JE}$ Jackson, ${ }^{3} \mathrm{CL}$ Shovlin. ${ }^{1} /$ mperial College School of Medicine, London, UK; ${ }^{2}$ Imperial College Hospital NHS Trust, London, UK; ${ }^{3}$ Imperial College London and Imperial College Hospital NHS Trust, London, UK

\subsection{6/thoraxjnl-2016-209333.178}

Introduction and objectives Individuals with pulmonary arteriovenous malformations (PAVMs), who often concurrently have hereditary haemorrhagic telangiectasia (HHT), are at increased risk of cerebral abscess, frequently resulting in life-changing deficits. Current aetiological consensus supports paradoxical emboli of periodontal origin, with preventative dental recommendations published in 2008. ${ }^{1}$ Limited knowledge is available to identify specifically at-risk individuals. This study aimed to characterise a cohort from 2005-2016; recognise potential precipitants; and compare results to an earlier published series.

Methods With ethical approval, notes of patients newly presenting at a single institution between 2005-2016 were reviewed to collect cohort characteristics. GraphPad Prism was used to calculate descriptive statistics, and to perform Mann-Whitney and Chisquared statistical tests for comparison between the two cohorts. Results Of 488 new patients with PAVMs, 33 (6.8\%) had cerebral abscesses. 21 were female (63.6\%), 12 male. The rate corrected for ascertainment bias was $3.8 \%$. The median age at abscess was 46 years (range 13-69). The median oxygen saturation $\left(\mathrm{SaO}_{2}\right)$ was $90.75 \%$ (range 70-97.5\%), with 9 individuals having respiratory symptoms. There were 29 confirmed HHT diagnoses (87.9\%). The median largest feeding artery diameter was $5 \mathrm{~mm}$, and for 5 individuals, all feeding arteries had diameter $\leq 3 \mathrm{~mm}$. In total, $19(57.6 \%)$ had residual PAVMs too small for embolization.

Organisms identified (Table 1) suggest periodontal origin; 16 individuals (48.5\%) had poor dental hygiene and 9 (27.3\%) had dental events as abscess precipitants. Interestingly, 4 individuals had abscesses whilst on holiday abroad. 5 individuals reported worsening migraines and 2 individuals had increased seizure frequency $\leq 3$ months pre-abscess.

Within the non-overlapping 1999-2005 cohort, there were 28/219 abscesses (12.8\%, 9.05\% adjusting for ascertainment bias). Compared to the later series, similar proportions of abscesses occurred prior to PAVM diagnosis (18/28 (64.3\%) vs. $24 / 33$ (72.3\%)). Proportionally more males were affected (57.1\%). No other significant differences were found for age, $\mathrm{SaO}_{2}$, feeding artery diameter, presence of respiratory symptoms, or HHT (all $\mathrm{p}$ values $>0.12$ ).

Conclusion The PAVM cohort remains at high risk of cerebral abscess. Scrupulous dental hygiene appears to remain paramount to reduce risk. The worsening migraines and abscess occurrence whilst abroad are unexpected findings recommended for further investigation. 
Abstract P35 Table 1 A list of the bacterial organisms that were cultured from cerebral abscesses in the 2005-2016 cohort

\begin{tabular}{|l|}
\hline \multicolumn{1}{|c|}{ Bacterial organisms from cerebral abscesses in PAVM patients } \\
\hline Actinomycosis spp \\
\hline Alpha haemolytic streptococci \\
Staphylococcus Intermedius \\
Streptococcus Milleri \\
\hline Streptococcus Anginosus \\
Actinomycosis Israelii \\
Streptococcus Constellatus \\
Non-haemolytic streptococci \\
Non specified streptococcal spp \\
\hline Non specified anaerobic species \\
\hline
\end{tabular}

\section{REFERENCE}

1 Shovlin C, et al. Post-NICE 2008: Antibiotic prophylaxis prior to dental procedures for patients with pulmonary arteriovenous malformations (PAVMs) and hereditary haemorrhagic telangiectasia. Br Dent J 2008; 205(10):531-3.

\section{P36 INJECTIONS OF INTRAVENOUS CONTRAST FOR COMPUTERISED TOMOGRAPHY SCANS PRECIPITATE MIGRAINES IN HEREDITARY HAEMORRHAGIC TELANGIECTASIA SUBJECTS AT RISK OF PARADOXICAL EMBOLI: IMPLICATIONS FOR RIGHT-TO-LEFT SHUNT RISKS}

${ }^{1}$ T Patel, ${ }^{2}$ A Elphick, ${ }^{3}$ JE Jackson, ${ }^{2} \mathrm{CL}$ Shovlin. ${ }^{1}$ St George's Hospital, London, UK; ${ }^{2}$ Imperial College London, London, UK; ${ }^{3}$ mperial College Healthcare NHS Trust, London, UK

\subsection{6/thoraxjnl-2016-209333.179}

Introduction and objectives Migraine headaches commonly affect people with pulmonary arteriovenous malformations (PAVMs) that provide right-to-left shunts. The majority of PAVMs are due to underlying hereditary haemorrhagic telangiectasia (HHT). In our clinical practice, patients occasionally reported acute precipitation of migraine headaches following injection of technetiumlabelled albumin macroaggregates for nuclear medicine scans. Our goal was to evaluate if injection of intravenous particles may provoke migraines in the cohort.

Methods Self-reported migraine features and exacerbations were examined in HHT subjects with and without pulmonary AVMs, for a series of noninvasive and invasive investigations, using an unbiased online survey. With ethical approval, the study recruited between August 2013-April 2015. Data were downloaded in December 2015 for analysis using STATA IC v12 and GraphPad Prism. Two group comparisons were performed using Mann Whitney or Fisher's exact test (for proportions). Multiple groups were compared using Kruskal Wallis with Dunn's multiple comparison test.

Results 166 subjects were classified as having both HHT and migraines. HHT subjects with migraines were more likely to have pulmonary AVMs $(\mathrm{p}<0.0001)$. Pulse oximetry, x-rays, ultrasound and computerised tomography (CT) scans without intravenous contrast medium rarely, if ever, provoked migraines, but unenhanced magnetic resonance imaging (MRI) was reported to exacerbate migraines by $14 / 124(11.2 \%)$ subjects. 114 had both enhanced and unenhanced CT examinations: studies with contrast media were more commonly reported to start $(9 / 114$ [7.8\%]), and/or worsen migraines (18/114 [15.7\%]) compared to those undertaken without contrast medium $(\mathrm{p}<0.01)$, or after simple blood tests $(\mathrm{p}<0.05)$. Additionally, migraine exacerbation was reported by $9 / 90(10 \%)$ after contrast echocardiography, $2 / 44(4.5 \%)$ after nuclear medicine scans, and 10/154 (6.5\%) after blood tests.

Conclusions In this population, MRI studies, blood tests, contrast echocardiograms, and intravenous injection of iodinated contrast medium associated with CT examinations were reported to provoke or exacerbate migraines. Since air emboli are recognised to complicate intravenous injections, particularly following pressurised pump injections of CT scan contrast, evaluation of migraines as a potential read-out for paradoxical emboli is recommended. In the meantime, for people with HHT and migraines, pre-test counselling may helpfully include advice to bring migraine preventers or treatments to help alleviate symptoms promptly.

\section{Imaginative Imaging in Lung Disease}

\section{P37 PRELIMINARY NORMAL VALUES FOR STRUCTURED LIGHT PLETHYSMOGRAPHY TIDAL BREATHING PARAMETERS AND AGE AND GENDER DIFFERENCES}

${ }^{1} S$ Fakhr, ${ }^{2} \mathrm{~L}$ O'Reilly, ${ }^{1} \mathrm{R}$ Wilson, ${ }^{2} \mathrm{~B}$ Cooper, ${ }^{3} \mathrm{R}$ Iles. ${ }^{1}$ PneumaCare Limited, Ely, UK; ${ }^{2}$ Queen Elizabeth Hospital, Birmingham, UK; ${ }^{3}$ Evelina Children's Hospital, London, UK

\subsection{6/thoraxjnl-2016-209333.180}

Introduction This is the first report from an ongoing study to define normal values for Structured Light Plethysmography (SLP) tidal breathing parameters in adults. Structured Light Plethysmography (SLP) is a non-contact, non-invasive respiratory measurement technology that utilises the movement of thoracoabdominal (TA) wall to measure a range of tidal breathing parameters. Various studies have been using SLP but lack of normative values can make any clinical judgement difficult.

Abstract P37 Table 1 SLP Tidal Breathing Parameters for adult male and female normals aged 18-69 years

\begin{tabular}{|c|c|c|c|c|c|c|c|}
\hline Parameter & $\begin{array}{l}\text { Males } 18-39 \text { yrs } \\
(\mathrm{n}=32) \text { Mean } \pm \text { SD }\end{array}$ & $\begin{array}{l}\text { Males } 40-69 \text { yrs } \\
(n=25) \text { Mean } \pm \text { SD }\end{array}$ & $\begin{array}{l}\text { Young vs older } \\
\text { males, } t(p)\end{array}$ & $\begin{array}{l}\text { Females } 18-39 \\
(\mathrm{n}=21) \text { Mean } \pm S D\end{array}$ & $\begin{array}{l}\text { Females } 40-69 \text { yrs } \\
(\mathrm{n}=29) \text { Mean } \pm \text { SD }\end{array}$ & $\begin{array}{l}\text { Young vs older } \\
\text { Females, } t(p)\end{array}$ & $\begin{array}{l}\text { Males vs. Females } \\
\text { (all ages), t (p) }\end{array}$ \\
\hline TAA & $5.7 \pm 23.3$ & $4.75 \pm 2.69$ & $1.18(0.24)$ & $4.85 \pm 2.45$ & $4.8 \pm 1.83$ & $0.08(0.94)$ & $0.92(0.36)$ \\
\hline LRHTA & $2.24 \pm 2.13$ & $2.39 \pm 1.64$ & $-0.298(0.77)$ & $1.58 \pm 0.69$ & $2.04 \pm 1.43$ & $-1.36(0.18)$ & $1.47(0.14)$ \\
\hline$\%$ RC & $45.87 \pm 13.07$ & $56.29 \pm 11.03$ & $-3.2(<0.01)$ & $60.23 \pm 8.55$ & $61.31 \pm 10.33$ & $-0.39(0.70)$ & $-4.62(<0.001)$ \\
\hline IE50 & $1.34 \pm 0.27$ & $1.25 \pm 0.18$ & $1.48(0.14)$ & $1.37 \pm 0.2$ & $1.42 \pm 0.29$ & $-0.64(0.52)$ & $-1.94(0.06)$ \\
\hline tPTEF/tE & $0.34 \pm 0.09$ & $0.26 \pm 0.07$ & $3.67(<0.001)$ & $0.32 \pm 0.09$ & $0.26 \pm 0.06$ & $2.62(<0.05)$ & $0.91(0.36)$ \\
\hline tPTIF/tl & $0.49 \pm 0.09$ & $0.55 \pm 0.09$ & $-2.69(<0.01)$ & $0.5 \pm 0.08$ & $0.52 \pm 0.07$ & $0.88(0.38)$ & $-1.13(0.26)$ \\
\hline
\end{tabular}

TAA: Thoraco-abdominal asynchrony (TAA), LRHTA:left vs Right Hemi-thoracic asynchrony, IE50:Inspiratory to expiratory flow at 50\% of tidal volume calculated from thoraco-abdominal wall displacement, tPTEF/tE: normalised time to reach peak tidal expiratory flow, tPTIF/tI: normalised time to reach peak tidal inspiratory flow 